\title{
Efficient quantum repeater with respect to both entanglement-concentration rate and complexity of local operations and classical communication
}

\author{
Zhaofeng $\mathrm{Su}^{*}$ \\ Centre for Quantum Software and Information, University of Technology Sydney, Ultimo, NSW 2007, Australia \\ and Institute of Computer Science Theory, School of Data and Computer Science, Sun Yat-Sen University, Guangzhou 510006, China \\ Ji Guan \\ Centre for Quantum Software and Information, University of Technology Sydney, Ultimo, NSW 2007, Australia \\ Lvzhou $\mathrm{Li}^{\dagger}$ \\ Institute of Computer Science Theory, School of Data and Computer Science, Sun Yat-Sen University, Guangzhou 510006, China
}

(Received 18 September 2017; published 22 January 2018)

\begin{abstract}
Quantum entanglement is an indispensable resource for many significant quantum information processing tasks. However, in practice, it is difficult to distribute quantum entanglement over a long distance, due to the absorption and noise in quantum channels. A solution to this challenge is a quantum repeater, which can extend the distance of entanglement distribution. In this scheme, the time consumption of classical communication and local operations takes an important place with respect to time efficiency. Motivated by this observation, we consider a basic quantum repeater scheme that focuses on not only the optimal rate of entanglement concentration but also the complexity of local operations and classical communication. First, we consider the case where two different two-qubit pure states are initially distributed in the scenario. We construct a protocol with the optimal entanglement-concentration rate and less consumption of local operations and classical communication. We also find a criterion for the projective measurements to achieve the optimal probability of creating a maximally entangled state between the two ends. Second, we consider the case in which two general pure states are prepared and general measurements are allowed. We get an upper bound on the probability for a successful measurement operation to produce a maximally entangled state without any further local operations.
\end{abstract}

DOI: 10.1103/PhysRevA.97.012325

\section{INTRODUCTION}

In the last three decades, quantum computation and quantum information have become one of the most active research fields. Many significant quantum information processing protocols have been proposed [1,2]. Remarkable progress has been achieved in both theoretical and experimental directions. For example, this includes quantum teleportation for sending an unknown quantum bit [3], quantum key distribution for quantum cryptography [4], and quantum dense coding for communicating two bits by sending only one qubit $[5,6]$. Note that quantum dense coding is somewhat like the inverse of quantum teleportation. As the most basic and counterintuitive characteristic of quantum mechanics, quantum entanglement plays an indispensable role in all the above applications.

The first step towards the implementation of these applications is to distribute quantum entanglement over remotely located participants. However, there are two bottlenecks to directly sending quantum states over a long distance [7]. On one hand, the probability of absorption when transmitting a photon increases exponentially with the distance. For example, a 1-km-long fiber has a transmission of $95 \%$, while the rate for a $1000-\mathrm{km}$ fiber is $10^{-10} \mathrm{~Hz}$, which means that a photon will be

\footnotetext{
"zhaofeng.su@student.uts.edu.au

†lilvzh@mail.sysu.edu.cn
}

transmitted successfully every 300 years [8]. On the other hand, even when a photon arrives at the destination, the fidelity of the transmitted state decreases exponentially with the distance because of the noise in quantum channels. Theoretical results also indicate that it is impossible to find a revised protocol to circumvent this rate-distance trade-off in the application of the quantum key distribution [9-12].

An effective solution to overcome the rate-distance trade-off is to use quantum repeaters to divide the long distance into many shorter segments. Each of the segments has a tolerable probability for absorption and noise. Since the first protocol was proposed by Briegel in 1998 [7], many schemes for realizing quantum repeaters have been developed [13-17]. Significant experimental progress has also been made in the last two decades [18-23]. The performance of quantum repeaters can be assessed by using bounds on the secret-key-agreement capacity of quantum communication channels [11].

In the basic scenario of quantum repeaters, two copies of a bipartite quantum state $\rho$ are shared by three participants, say Alice, Clare, and Bob. Alice and Clare share the copy $\rho_{A C}$, and Clare and Bob share $\rho_{C B}$. By performing local operations and classical communication (LOCC) between these three parties, quantum entanglement can be created between Alice and Bob, thereby extending the distance of the entanglement distribution [8]. The basic scenario is depicted in Fig 1.

This basic scenario of quantum repeaters is also known as quantum entanglement swapping [24]. When less entangled 


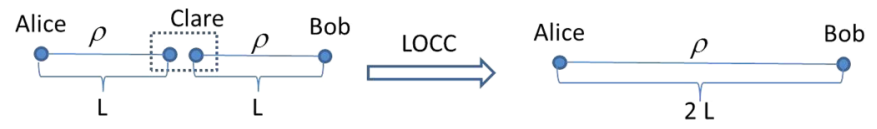

FIG. 1. Basic scenario of quantum repeaters.

pure states are initially distributed in the scenario, the process of concentrating a maximally entangled state is termed entanglement concentration [25,26]. A simpler scenario is one with only two participants, which has been extensively studied [6,27-29]. Many results have also been achieved for quantum entanglement swapping [30-34]. Bose et al. considered the case where the same two-qubit pure states were prepared in each segment [30]. They found the optimal strategy of quantum swapping with respect to concentrating the most entanglement between Alice and Bob. Shi et al. considered the case where different two-qubit pure states were prepared [31]. In their strategy, Clare performs a projective measurement in the standard Bell basis. Then, Alice and Bob perform local operations to create a maximally entangled state between them. Shi et al. found out that the optimal entanglement-concentration rate was exactly the same as the concentration rate in the scenario where the less entangled single resource was directly distributed between Alice and Bob. Hardy and Song considered the case where general entangled pure states were distributed in a chain scenario [32]. Perseguers et al. exploited the scenario where general two-qubit pure states were distributed in a quantum network [33]. The quantum entanglement swapping configuration can also be used to activate nonlocality from local resources [34]. We have considered a similar scenario for generating tripartite genuine nonlocality from bipartite nonlocal resources [35].

In the basic quantum repeater scheme, the protocol consists of the preparation of quantum resources and LOCC. In practice, quantum resources can be efficiently transmitted over a short distance [8]. Once the quantum resources are prepared, entanglement can be concentrated from the scenario by applying LOCC. Therefore, it is of practical significance to consider the LOCC complexity in the protocol. However, LOCC is considered an unlimited resource in the aforementioned references.

In this work, we present some basic quantum repeater schemes while considering both the entanglementconcentration rate and the LOCC complexity. First, we exploit the scenario where two different two-qubit pure states are prepared. We construct a protocol which can create maximal entanglement between Alice and Bob at the optimal rate and with less consumption of LOCC resources. We also find a criteria for the projective measurements which are able to achieve the optimal rate of entanglement concentration. Second, we consider the case in which two general pure states are prepared and general measurements are allowed. We get an upper bound on the probability for a successful measurement operation to produce a maximally entangled state between Alice and Bob such that the rate of entanglement concentration is as high as possible while the LOCC complexity is as low as possible.

\section{TAKING DIFFERENT TWO-QUBIT PURE STATES AS RESOURCES}

In this section, we consider the case where two different pure states of a two-qubit system are prepared in the basic quantum

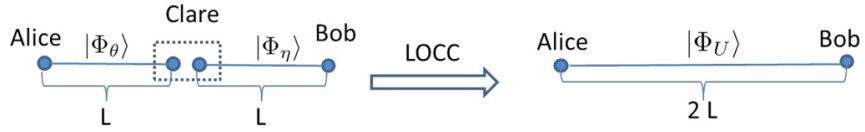

FIG. 2. Quantum repeater scheme with different two-qubit pure states.

repeater configuration. We construct a protocol to concentrate the maximally entangled two-qubit state between Alice and Bob at the optimal entanglement-concentration rate and with less LOCC complexity.

Up to some local unitary operations, any pure state of a two-qubit system can be written as $\left|\Phi_{\theta}\right\rangle \equiv \cos \theta|00\rangle+$ $\sin \theta|11\rangle, \theta \in\left[0, \frac{\pi}{2}\right]$. The state $\left|\Phi_{\theta}\right\rangle$ is said to be entangled if $\theta \in\left(0, \frac{\pi}{2}\right)$. Otherwise, it is separable. When $\theta=\frac{\pi}{4}$, the state is known as the standard maximally entangled state of a two-qubit system, denoted $|\Phi\rangle=(|00\rangle+|11\rangle) / \sqrt{2}$. Note that quantum entanglement is a kind of resource that cannot be created or increased with LOCC. Particularly, local unitary operations cannot affect the amount of entanglement that exists within entangled quantum systems. Thus, we only need to consider the case where the entangled states $\left|\Phi_{\theta}\right\rangle$ and $\left|\Phi_{\eta}\right\rangle$ with $\theta, \eta \in\left(0, \frac{\pi}{2}\right)$ are initially distributed in the configuration. A general two-qubit maximally entangled state is equivalent to $|\Phi\rangle$ up to a local unitary $U$, denoted as $\left|\Phi_{U}\right\rangle \equiv(U \otimes I)|\Phi\rangle$. By applying LOCC to the configuration, we expect to obtain a maximally entangled state $\left|\Phi_{U}\right\rangle$ between Alice and Bob such that the rate of entanglement concentration is as high as possible while the LOCC complexity is as low as possible. The configuration is depicted in Fig. 2. To achieve this goal, we first need to present some lemmas.

Lemma 1. Suppose two parties share a two-qubit state $\left|\Phi_{\lambda}\right\rangle=\cos \lambda|00\rangle+\sin \lambda|11\rangle$ with $\lambda \in\left(0, \frac{\pi}{2}\right)$. By performing LOCC, the state can be probabilistically transformed into a maximally entangled state. The probability of a successful transformation is upper bounded by $P_{E}\left(\Phi_{\lambda}\right) \equiv$ $\min \left\{2 \cos ^{2} \lambda, 2 \sin ^{2} \lambda\right\}$.

Lemma 1, which is an implication of Vidal's result [27], gives the upper bound on the entanglement-concentration rate in the scenario where an entangled two-qubit state $\left|\Phi_{\lambda}\right\rangle$ is shared by two parties. Without loss of generality, suppose $\cos \lambda \geqslant \sin \lambda$. The upper bound of the concentration rate can be obtained by performing a general measurement $\left\{M_{0}, M_{1}\right\}$, where $M_{0}=\tan \lambda|0\rangle\langle 0|+| 1\rangle\langle 1|$ and $M_{1}=\sqrt{1-\tan ^{2} \lambda}|0\rangle\langle 0|$, on either party. If the measurement outcome 0 is observed, the maximally entangled state will be created between them. The corresponding probability is $P_{E}\left(\Phi_{\lambda}\right)=2 \sin ^{2} \lambda$.

Applying Lemma 1, we get the upper bound on the entanglement-concentration rate for the simplest quantum repeater scenario depicted in Fig. 2. The result is concluded in the following lemma.

Lemma 2. Suppose the two-qubit states $\left|\Phi_{\theta}\right\rangle$ and $\left|\Phi_{\eta}\right\rangle$ are initially distributed in the scenario depicted in Fig. 2. Let $P_{\mathrm{MS}}$ be the optimal probability that the maximally entangled state can be created between Alice and Bob by applying LOCC. Then $P_{\mathrm{MS}} \leqslant \min \left\{P_{E}\left(\Phi_{\theta}\right), P_{E}\left(\Phi_{\eta}\right)\right\}$.

Proof. We prove this lemma via deducing contradictions. Suppose $P_{\mathrm{MS}}>P_{E}\left(\Phi_{\theta}\right)$. Then consider a scenario where the 
resource $\left|\Phi_{\theta}\right\rangle$ is shared by Alice and Bob. Let Bob locally prepare an ancilla state $\left|\Phi_{\eta}\right\rangle$. According to the definition, the probability of creating a maximally entangled state between Alice and Bob by applying LOCC is $P_{\mathrm{MS}}$, which is greater than $P_{E}\left(\Phi_{\theta}\right)$. This result contradicts Lemma 1 . Thus, the assumption is not true. It should have $P_{\mathrm{MS}} \leqslant P_{E}\left(\Phi_{\theta}\right)$. Similarly, we can get $P_{\mathrm{MS}} \leqslant P_{E}\left(\Phi_{\eta}\right)$. Therefore, we have $P_{\mathrm{MS}} \leqslant \min \left\{P_{E}\left(\Phi_{\theta}\right), P_{E}\left(\Phi_{\eta}\right)\right\}$

In our protocol, we consider a projective measurement on Clare's joint system. A successful projection of Clare is one where the maximally entangled state can be directly created between Alice and Bob without any further local operations. Our strategy is to construct a projective measurement such that the sum of the probabilities of the successful projections is as high as possible. For projections that result in Alice and Bob sharing nonmaximally entangled states, we can apply the probabilistic entanglement concentration by performing a local measurement operation on Bob's system. In general, we can concentrate entanglement from the scenario at a high rate while less of the LOCC resource is consumed.

In the following lemma, we work out the lower and upper bounds on the probability of a successful projection.

Lemma 3. Assume that the two-qubit pure states $\left|\Phi_{\theta}\right\rangle$ and $\left|\Phi_{\eta}\right\rangle$ are initially distributed in the scenario shown in Fig. 2. Without loss of generality, suppose $\theta, \eta \in\left(0, \frac{\pi}{4}\right]$. A maximally entangled state can be created between Alice and Bob by projecting Clare's joint system onto state $|\varphi\rangle$ without any further local operations. The probability $p(\varphi)$ of the successful projection is bounded by

$$
\frac{\sin ^{2} 2 \theta \sin ^{2} 2 \eta}{4(1+\cos 2 \theta \cos 2 \eta)} \leqslant p(\varphi) \leqslant \frac{\sin ^{2} 2 \theta \sin ^{2} 2 \eta}{4(1-\cos 2 \theta \cos 2 \eta)}
$$

Proof. The initial state of the three participants' joint quantum system can be written as

$$
\left|\phi_{0}\right\rangle_{A B C}=\sum_{k=0}^{3} f_{k}|k\rangle_{A B}|k\rangle_{C}
$$

where $f_{0}=\cos \theta \cos \eta, f_{1}=\cos \theta \sin \eta, f_{2}=\sin \theta \cos \eta$, and $f_{3}=\sin \theta \sin \eta$. Suppose $|\varphi\rangle=\sum_{k=0}^{3} \mu_{k}|k\rangle \in \mathcal{H}_{2}^{\otimes 2}$ with the constraint $\sum_{k=0}^{3}\left|\mu_{k}\right|^{2}=1$. Once the projection occurred, the state of Alice and Bob's joint system would be

$$
|\phi\rangle_{A B}=\frac{1}{\sqrt{p(\varphi)}}\left\langle\phi_{C} \mid \phi_{0}\right\rangle_{A B C}=\frac{1}{\sqrt{p(\varphi)}} \sum_{k=0}^{3} f_{k} \mu_{k}^{*}|k\rangle_{A B} .
$$

As we expect that a maximally entangled state would be created between Alice and Bob without any further local operations, it should have

$$
|\phi\rangle_{A B}=(U \otimes I)|\Phi\rangle,
$$

where $U$ is a unitary operator on $\mathcal{H}_{2}$.

Let $\Delta_{k} \equiv\langle k|(U \otimes I)| \Phi\rangle$. Then the parameters of the projection state and the corresponding probability are related by the formula as follows:

$$
f_{k} \mu_{k}^{*}=\sqrt{p(\varphi)} \Delta_{k} .
$$

By applying the unit constraint of the projection state $|\varphi\rangle$, we get the probability of the successful projection as follows:

$$
p(\varphi)=\left(\sum_{k=0}^{3} \frac{\left|\Delta_{k}\right|^{2}}{f_{k}^{2}}\right)^{-1}
$$

Without loss of generality, let $U=|0\rangle\left\langle\alpha_{0}|+| 1\right\rangle\left\langle\alpha_{1}\right|$, where $\left|\alpha_{0}\right\rangle=e^{i \tau_{0}}\left(\cos \alpha|0\rangle+e^{i \gamma} \sin \alpha|1\rangle\right)$ and $\left|\alpha_{1}\right\rangle=e^{i \tau_{1}}(\sin \alpha|0\rangle-$ $\left.e^{i \gamma} \cos \alpha|1\rangle\right)$ with $\alpha, \tau_{0}, \tau_{1}, \gamma \in[0,2 \pi)$. It is trivial to determine that $\left|\Delta_{0}\right|^{2}=\left|\Delta_{3}\right|^{2}=\frac{1}{2} \cos ^{2} \alpha$ and $\left|\Delta_{1}\right|^{2}=\left|\Delta_{2}\right|^{2}=\frac{1}{2} \sin ^{2} \alpha$. Hence, any successful projection state $|\varphi\rangle$ should be equivalently written as

$$
\begin{aligned}
|\varphi\rangle= & \sqrt{\frac{p(\varphi)}{2}}\left[\cos \alpha\left(\frac{1}{f_{0}}|00\rangle+e^{i \beta} \frac{1}{f_{3}}|11\rangle\right)\right. \\
& \left.+e^{i \beta^{\prime}} \sin \alpha\left(\frac{1}{f_{1}}|01\rangle+e^{i \beta^{\prime \prime}} \frac{1}{f_{2}}|10\rangle\right)\right],
\end{aligned}
$$

where $\alpha, \beta, \beta^{\prime}, \beta^{\prime \prime} \in[0,2 \pi)$. The inverse of the probability can be rewritten as

$$
\begin{aligned}
p(\varphi)^{-1}= & \frac{1}{2}\left(\frac{1}{f_{1}^{2}}+\frac{1}{f_{2}^{2}}\right) \\
& +\frac{1}{2} \cos ^{2} \alpha\left(\frac{1}{f_{0}^{2}}+\frac{1}{f_{3}^{2}}-\frac{1}{f_{1}^{2}}-\frac{1}{f_{2}^{2}}\right) .
\end{aligned}
$$

As we have assumed that $\theta, \eta \in\left(0, \frac{\pi}{4}\right]$, it follows that

$$
\frac{1}{f_{0}^{2}}+\frac{1}{f_{3}^{2}}-\frac{1}{f_{1}^{2}}-\frac{1}{f_{2}^{2}}=\frac{16 \cos 2 \theta \cos 2 \eta}{\sin ^{2} 2 \theta \sin ^{2} 2 \eta} \geqslant 0 .
$$

Hence, the inverse of the successful projection probability $p(\varphi)^{-1}$ reaches the maximum when $\cos ^{2} \alpha=1$ while it reaches the minimum when $\cos ^{2} \alpha=0$. Namely,

$$
p(\varphi)^{-1} \leqslant \frac{1}{2}\left(\frac{1}{f_{0}^{2}}+\frac{1}{f_{3}^{2}}\right)=\frac{4(1+\cos 2 \theta \cos 2 \eta)}{\sin ^{2} 2 \theta \sin ^{2} 2 \eta}
$$

and

$$
p(\varphi)^{-1} \geqslant \frac{1}{2}\left(\frac{1}{f_{1}^{2}}+\frac{1}{f_{2}^{2}}\right)=\frac{4(1-\cos 2 \theta \cos 2 \eta)}{\sin ^{2} 2 \theta \sin ^{2} 2 \eta} .
$$

Then Eq. (1) follows Eqs. (7) and (8) immediately. Therefore, we have proved the lemma.

Suppose Clare performs a projective measurement in the orthonormal basis $\left\{\left|\varphi_{k}\right\rangle\right\}_{k=1}^{4}$, with $p\left(\varphi_{k}\right)$ being the probability of projecting the system into state $\left|\varphi_{k}\right\rangle$ and $\left|\phi_{k}\right\rangle_{A B}$ being the corresponding postmeasurement state of Alice and Bob's joint system.

First, let $\left|\varphi_{1}\right\rangle$ be the successful projection such that $p\left(\varphi_{1}\right)$ reaches the upper bound of the projection probability in Eq. (1). The condition of achieving the upper bound probability is $\cos \alpha=0$ in Eq. (6). Thus, the projection state can be written as

$$
\left|\varphi_{1}\right\rangle=\left(f_{2}|01\rangle+e^{i \beta_{1}} f_{1}|10\rangle\right) / \sqrt{f_{1}^{2}+f_{2}^{2}}
$$

for an arbitrary phase $\beta_{1} \in[0,2 \pi)$. The corresponding projection probability is $p\left(\varphi_{1}\right)=\frac{\sin ^{2} 2 \theta \sin ^{2} 2 \eta}{4(1-\cos 2 \theta \cos 2 \eta)}$.

Second, we try to construct another successful projection state $\left|\varphi_{2}\right\rangle$. State $\left|\varphi_{2}\right\rangle$ should be of the form of Eq. (6). The 
orthogonality of the projection states $\left|\varphi_{2}\right\rangle$ and $\left|\varphi_{1}\right\rangle$ requires that $\left\langle\varphi_{1} \mid \varphi_{2}\right\rangle=0$, which is equivalent to the following constraint:

$$
\sqrt{\frac{p(\varphi)}{2\left(f_{1}^{2}+f_{2}^{2}\right)}} e^{i \beta^{\prime}} \sin \alpha\left(\frac{f_{2}}{f_{1}}+e^{i\left(\beta^{\prime \prime}-\beta_{1}\right)} \frac{f_{1}}{f_{2}}\right)=0 .
$$

To let the above constraint hold for general resources $\left|\Phi_{\theta}\right\rangle$ and $\left|\Phi_{\eta}\right\rangle$, it should have $\sin \alpha=0$. Thus, we get the second projection state as

$$
\left|\varphi_{2}\right\rangle=\left(f_{3}|00\rangle+e^{i \beta_{2}} f_{0}|11\rangle\right) / \sqrt{f_{0}^{2}+f_{3}^{2}},
$$

where $\beta_{2} \in[0,2 \pi)$ is an arbitrary phase. The corresponding probability of the successful projection is $p\left(\varphi_{2}\right)=$ $\frac{\sin ^{2} 2 \theta \sin ^{2} 2 \eta}{4(1+\cos 2 \theta \cos 2 \eta)}$, which is exactly the lower bound of the successful probability.

A simple calculation shows that there is not a third projection state which is of the form of Eq. (6) and orthogonal to the projection states $\left|\varphi_{1}\right\rangle$ and $\left|\varphi_{2}\right\rangle$. The other projection states can be chosen as

$$
\begin{aligned}
& \left|\varphi_{3}\right\rangle=\left(f_{1}|01\rangle-e^{i \beta_{1}} f_{2}|10\rangle\right) / \sqrt{f_{1}^{2}+f_{2}^{2}}, \\
& \left|\varphi_{4}\right\rangle=\left(f_{0}|00\rangle-e^{i \beta_{2}} f_{3}|11\rangle\right) / \sqrt{f_{0}^{2}+f_{3}^{2}},
\end{aligned}
$$

with the projection probabilities being $p\left(\varphi_{3}\right)=\frac{f_{1}^{4}+f_{2}^{4}}{f_{1}^{2}+f_{2}^{2}}$ and $p\left(\varphi_{4}\right)=\frac{f_{0}^{4}+f_{3}^{4}}{f_{0}^{2}+f_{3}^{2}}$, respectively. The corresponding postmeasurement states of Alice and Bob's joint system are

$$
\begin{aligned}
\left|\phi_{3}\right\rangle_{A B} & =\left(f_{1}^{2}|01\rangle-e^{-i \beta_{1}} f_{2}^{2}|10\rangle\right) / \sqrt{f_{1}^{4}+f_{2}^{4}}, \\
\left|\phi_{4}\right\rangle_{A B} & =\left(f_{0}^{2}|00\rangle-e^{-i \beta_{2}} f_{3}^{2}|11\rangle\right) / \sqrt{f_{0}^{4}+f_{3}^{4}} .
\end{aligned}
$$

We have figured out a projective measurement for Clare. Two of the projections would directly leave Alice and Bob's joint system in maximally entangled states, while the others would leave them in less entangled states. When the measurement outcome $\left|\varphi_{3}\right\rangle$ or $\left|\varphi_{4}\right\rangle$ is observed, we can still concentrate some entanglement from the less entangled state $\left|\phi_{3}\right\rangle$ or $\left|\phi_{4}\right\rangle$ by performing local operations on either Alice's or Bob's system.

Let $q$ and $q^{\prime}$ be the probabilities that we can concentrate the maximally entangled state from $\left|\phi_{3}\right\rangle$ and $\left|\phi_{4}\right\rangle$ by performing the corresponding general measurement on Bob's qubit, respectively. As we have assumed that $\theta, \eta \in\left(0, \frac{\pi}{4}\right]$, it follows that $f_{0} \geqslant f_{3}$. Without loss of generality, we suppose $\eta \geqslant \theta$, which implies that $f_{1} \geqslant f_{2}$. Applying Lemma 1 , we get

$$
q=\frac{2 f_{2}^{4}}{f_{1}^{4}+f_{2}^{4}}, \quad q^{\prime}=\frac{2 f_{3}^{4}}{f_{0}^{4}+f_{3}^{4}} .
$$

In general, the maximally entangled resource can be created between Alice and Bob with probability

$$
P_{\mathrm{MS}}=p\left(\varphi_{1}\right)+p\left(\varphi_{2}\right)+p\left(\varphi_{3}\right) q+p\left(\varphi_{4}\right) q^{\prime}=2 \sin ^{2} \theta .
$$

Note that $2 \sin ^{2} \theta$ also equals the optimal probability $P_{E}\left(\Phi_{\theta}\right)$ that the maximally entangled state can be concentrated in the scenario where the resource $\left|\Phi_{\theta}\right\rangle$ is directly prepared between Alice and Bob. Thus, we have $P_{\mathrm{MS}}=P_{E}\left(\Phi_{\theta}\right)$.
Similarly, we get $P_{\mathrm{MS}}=P_{E}\left(\Phi_{\eta}\right)$ for the case $\theta \geqslant$ $\eta$. Thus, the maximally entangled resource can be concentrated from the scenario in Fig. 2 with probability $P_{\mathrm{MS}}=\min \left\{P_{E}\left(\Phi_{\theta}\right), P_{E}\left(\Phi_{\eta}\right)\right\}$. According to Lemma 2, $P_{\mathrm{MS}} \leqslant \min \left\{P_{E}\left(\Phi_{\theta}\right), P_{E}\left(\Phi_{\eta}\right)\right\}$. Therefore, the entanglementconcentration rate obtained in our protocol is the optimal one for the scenario.

Upon performing the projective measurement on Clare's joint system, no further local operation is needed when any of the two successful projections is observed. Maximally entangled resources will be directly created between Alice and Bob with probability $\frac{\sin ^{2} 2 \theta \sin ^{2} 2 \eta}{2\left(1-\cos ^{2} 2 \theta \cos ^{2} 2 \eta\right)}$. Assume that the classical communication channel is liable. It is possible to establish an efficient classical communication agreement for telling Bob to take the corresponding action.

Therefore, we have constructed a protocol for extending the distance of the entanglement distribution, which is optimal with respect to the entanglement-concentration rate and efficient in perspective LOCC complexity. With this, we have proved the following theorem, which is the main result of this section.

Theorem 1. Suppose two different pure states $\left|\Phi_{\theta}\right\rangle$ and $\left|\Phi_{\eta}\right\rangle$ of a two-qubit system are initially distributed in the scenario shown in Fig. 2. We construct a protocol as follows. First, apply a projective measurement in the orthonormal basis $\left\{\mid \varphi_{k}\right\}_{k=1}^{4}$, which is defined in Eqs. (9)-(12), to Clare's joint system. Second, selectively perform a local operation on Bob's system according to Clare's measurement outcome. Then a maximally entangled resource could be created between Alice and Bob with probability $P_{\mathrm{MS}}=\min \left\{P_{E}\left(\Phi_{\theta}\right), P_{E}\left(\Phi_{\eta}\right)\right\}$. This protocol is optimal for perspectives of entanglement-concentration rate and efficient for perspectives of LOCC complexity.

\section{A. Criterion for optimal projective measurements with respect to the entanglement-concentration rate}

For the scenario depicted in Fig. 2, the projective measurement in the standard Bell basis is also able to achieve the optimal entanglement-concentration rate [31]. Thus, the optimal projective measurement for Clare's joint system is not unique with respect to the entanglement-concentration rate. However, not all projective measurements can achieve that goal. For example, it is impossible to obtain entanglement between Alice and Bob by projecting Clare's system onto separable states.

Hence, it is a practical problem to verify whether a projective measurement is able to concentrate entanglement from the scenario with the optimal rate. The following theorem gives a criterion for such measurements.

Theorem 2. We consider a scenario with states $\left|\Phi_{\theta}\right\rangle$ and $\left|\Phi_{\eta}\right\rangle$, which is shown in Fig. 2. Without loss of generality, suppose $\theta, \eta \in\left(0, \frac{\pi}{4}\right]$ and $\theta \leqslant \eta$. Suppose $P \equiv\left\{P_{k}\right\}_{k=1}^{4}$ is a projective measurement on $\mathcal{H}_{2}^{\otimes 2}$ where $P_{k}$ are projectors. With the assistance of LOCC, the projective measurement $P$ on Clare's joint system is able to achieve the optimal entanglement-concentration rate if and only if

$$
\begin{aligned}
& \sum_{k=1}^{4} \sqrt{\left[\operatorname{tr}\left(T_{1} \otimes T_{2} P_{k}\right)\right]^{2}+\sin ^{2} 2 \theta\left|\operatorname{tr}\left(|0\rangle\langle 1| \otimes T_{2} P_{k}\right)\right|^{2}} \\
& \quad=\cos 2 \theta
\end{aligned}
$$


where the operators $T_{1}=\cos ^{2} \theta|0\rangle\left\langle 0\left|-\sin ^{2} \theta\right| 1\right\rangle\langle 1|$ and $T_{2}=$ $\cos ^{2} \eta|0\rangle\left\langle 0\left|+\sin ^{2} \eta\right| 1\right\rangle\langle 1|$.

Proof. Suppose $P_{k}=\left|\varphi_{k}\right\rangle\left\langle\varphi_{k}\right|$, where $\left\{\left|\varphi_{k}\right\rangle\right\}_{k=1}^{4}$ is an orthonormal basis of the space $\mathcal{H}_{2}^{\otimes 2}$. Note that the initial state of the configuration is $\left|\Phi_{\theta}, \Phi_{\eta}\right\rangle=\sum_{t=0}^{3} f_{t}|t\rangle_{C}|t\rangle_{A B}$. In the case where the measurement outcome $k$ is observed, the postmeasurement state of Alice and Bob's joint system will be

$$
\left|\phi_{k}\right\rangle_{A B}=\frac{1}{\sqrt{p_{k}}} \sum_{t=0}^{3} f_{t}\left\langle\varphi_{k} \mid t\right\rangle|t\rangle_{A B},
$$

where $p_{k}$ is the probability of observing the measurement result $k$. From Lemma 1 , the probability $P_{E}\left(\phi_{k}\right)$ is twice the square of the state's minimal Schmidt number. It is also equal to twice the minimal eigenvalue of the density operator of either Alice's or Bob's system. On the condition that Alice and Bob share the state $\left|\phi_{k}\right\rangle_{A B}$, the density operator of Alice's system is

$$
\rho_{A}^{k} \equiv \operatorname{tr}_{B}\left(\left|\phi_{k}\right\rangle_{A B}\left\langle\phi_{k}\right|\right)=\frac{1}{p_{k}} \sum_{t_{1}, t_{2}=0}^{1} a_{t_{1} t_{2}}^{k}\left|t_{1}\right\rangle\left\langle t_{2}\right|,
$$

where $a_{t_{1} t_{2}}^{k}=\theta_{t_{1}} \theta_{t_{2}} \operatorname{tr}\left(\left(\left|t_{1}\right\rangle\left\langle t_{2}\right| \otimes T_{2}\right) P_{k}\right)$. For the purpose of convenience, we take the notation $\theta_{0} \equiv \cos \theta$ and $\theta_{1} \equiv \sin \theta$. The eigenvalues of $\rho_{A}^{k}$ are

$$
\lambda_{ \pm}^{k}=\frac{1}{2 p_{k}}\left[\left(a_{00}^{k}+a_{11}^{k}\right) \pm \sqrt{\left(a_{00}^{k}-a_{11}^{k}\right)^{2}+4 a_{01}^{k} a_{10}^{k}}\right] .
$$

As $\lambda_{+}^{k} \geqslant \lambda_{-}^{k}$, we get $P_{E}\left(\phi_{k}\right)=2 \lambda_{-}^{k}$.

When all the measurement outcomes are considered, the maximally entangled state can be concentrated from the scenario at rate

$$
\begin{aligned}
p_{s} & \equiv \sum_{k=1}^{4} p_{k} P_{E}\left(\phi_{k}\right) \\
& =\sum_{k=1}^{4}\left[\left(a_{00}^{k}+a_{11}^{k}\right)-\sqrt{\left(a_{00}^{k}-a_{11}^{k}\right)^{2}+4 a_{01}^{k} a_{10}^{k}}\right] .
\end{aligned}
$$

Note $\quad$ that $\quad \sum_{k=1}^{4} a_{00}^{k}=\sum_{k=1}^{4} \cos ^{2} \theta \operatorname{tr}\left[\left(|0\rangle\langle 0| \otimes T_{2}\right) P_{k}\right]=$ $\cos ^{2} \theta$. Similarly, we get $\sum_{k=1}^{4} a_{11}^{k}=\sin ^{2} \theta$. It is trivial to see that $a_{00}^{k}-a_{11}^{k}=\operatorname{tr}\left[\left(T_{1} \otimes T_{2}\right) P_{k}\right]$ and $4 a_{01}^{k} a_{10}^{k}=$ $\sin ^{2} 2 \theta\left|\operatorname{tr}\left(\left(|0\rangle\langle 1| \otimes T_{2}\right) P_{k}\right)\right|^{2}$. Thus, the rate can be equivalently written as

$$
\begin{aligned}
p_{s}= & 1 \\
& -\sum_{k=1}^{4} \sqrt{\left\{\operatorname{tr}\left[\left(T_{1} \otimes T_{2}\right) P_{k}\right]\right\}^{2}+\sin ^{2} 2 \theta\left|\operatorname{tr}\left(|0\rangle\langle 1| \otimes T_{2}\right) P_{k}\right|^{2}} .
\end{aligned}
$$

According to Theorem 1, the optimal entanglementconcentration rate of the scenario shown in Fig. 2 is $2 \sin ^{2} \theta$. Therefore, the projective measurement $P$ is able to achieve the optimal entanglement-concentration rate, which means $p_{s}=2 \sin ^{2} \theta$, if and only if

$$
\begin{aligned}
& \sum_{k=1}^{4} \sqrt{\left[\operatorname{tr}\left(T_{1} \otimes T_{2} P_{k}\right)\right]^{2}+\sin ^{2} 2 \theta\left|\operatorname{tr}\left(|0\rangle\langle 1| \otimes T_{2} P_{k}\right)\right|^{2}} \\
& \quad=\cos 2 \theta .
\end{aligned}
$$

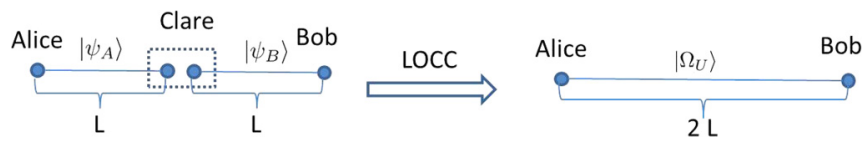

FIG. 3. Quantum repeater scenario with two general pure states.

Simple calculations show that both the projective measurement in the standard Bell basis and the one we proposed in this section fulfill the criterion.

\section{UPPER BOUND FOR THE SCENARIO WITH DIFFERENT GENERAL PURE STATES}

In this section, we consider a more general scenario in which different general pure states are prepared and general measurements are allowed. Suppose a general bipartite pure state $\left|\psi_{A B}\right\rangle$ is shared by Alice and Clare, while $\left|\psi_{C B}\right\rangle$ is shared by Clare and Bob. We analyze the measurement outcome which leaves Alice and Bob in a maximally entangled state without any further local operations. We refer to this measurement outcome as a successful measurement outcome. The scenario is depicted in Fig. 3. Note that an arbitrary maximally entangled state can be written as $\left|\Omega_{U}\right\rangle \equiv(U \otimes I)|\Omega\rangle$, where $U$ is a local unitary on $\mathcal{H}_{d}$ and $|\Omega\rangle=\frac{1}{\sqrt{d}} \sum_{k=1}^{d}|k\rangle|k\rangle$ is the standard maximally entangled state.

The probability of observing a successful measurement outcome varies for different $U$ 's. Theorem 3 gives an upper bound on the probability, which is the main result in this section. To prove Theorem 3, we need two lemmas. Lemma 4 is concluded from Wolf's lecture notes [36]. Lemma 5 is a generalization of a mathematical theorem which has been proved by Lewis [37]. We denote $\lambda_{\downarrow}(A)$ for the column vector of operator $A$ 's eigenvalues in nonincreasing order and $\lambda_{\uparrow}(A)$ for that in nondecreasing order.

Lemma 4. Suppose $|\psi\rangle$ is a bipartite pure state of the joint quantum system $A \otimes B$. Let $\rho_{A} \equiv \operatorname{tr}_{B}(|\psi\rangle\langle\psi|)$ be the density operator of subsystem $A$. Suppose $\rho_{A}$ can be expressed as a convex combination $\rho_{A}=\sum_{i} \lambda_{i} \rho_{i}$, where $\lambda_{i}>0, \sum_{i} \lambda_{i}=1$ and $\rho_{i}$ are density operators on $\mathcal{H}_{A}$. Then there is a quantum measurement operation on system $B$, say $T=\left\{T_{i}: \mathcal{B}\left(\mathcal{H}_{\mathcal{B}}\right) \rightarrow\right.$ $\left.\mathcal{B}\left(\mathcal{H}_{\mathcal{B}}\right)\right\}$, such that

$$
\lambda_{i} \rho_{i}=\operatorname{tr}_{B}\left[\left(I \otimes T_{i}\right)(|\psi\rangle\langle\psi|)\right] .
$$

The parameter $\lambda_{i}$ can be interpreted as the probability of observing measurement outcome $i$. The upper bound of $\lambda_{i}$ is as follows:

$$
\lambda_{i} \leqslant\left\|\rho^{-\frac{1}{2}} \rho_{i} \rho^{-\frac{1}{2}}\right\|_{\infty}^{-1} .
$$

Lemma 5. For Hermitian operators $A$ and $B$,

$$
\operatorname{tr}(A B) \geqslant \lambda_{\uparrow}(A)^{T} \lambda_{\downarrow}(B)
$$

with equality if and only if there is a unitary operator $U$ such that $U^{\dagger} A U=\operatorname{diag}\left[\lambda_{\uparrow}(A)\right]$ and $U^{\dagger} B U=\operatorname{diag}\left[\lambda_{\downarrow}(B)\right]$.

Theorem 3. Suppose two general pure states $\left|\psi_{A C}\right\rangle=$ $\sum_{k=1}^{d_{A}} \sqrt{a_{k}}|k\rangle|k\rangle$ and $\left|\psi_{C B}\right\rangle=\sum_{k=1}^{d_{B}} \sqrt{b_{k}}|k\rangle|k\rangle$ are prepared in the scenario, which is shown in Fig. 3. Without loss of generality, suppose $a_{1} \geqslant a_{2} \geqslant \ldots a_{d_{A}}>0, b_{1} \geqslant b_{2} \geqslant \ldots b_{d_{B}}>0$ and $d \equiv d_{B} \geqslant d_{A}$. Clare performs a general measurement $M \equiv\left\{M_{i}\right\}$ on his joint system, with $p_{i}$ being the probability 
of observing measurement outcome $i$. Suppose Alice and Bob will share a maximally entangled state $\left|\Omega_{U}\right\rangle \in \mathcal{H}_{d}^{\otimes 2}$ when the measurement outcome $i$ is observed by Clare. Then the corresponding probability $p_{i}$ has the following upper bound:

$$
p_{i} \leqslant \frac{d}{\sum_{k=1}^{d_{A}} \frac{1}{a_{k} b_{d_{A}+1-k}}} \equiv p_{\max } .
$$

The upper bound can be achieved by setting the measurement operator $M_{i}=\sqrt{p_{\max }}\left|\Omega_{U}\right\rangle\left\langle\Omega_{U}\right| \rho_{A B}^{-\frac{1}{2}}$, where $U=$ $\sum_{k=1}^{d_{A}}\left|d_{A}+1-k\right\rangle\left\langle k\left|+\sum_{k=d_{A}+1}^{d}\right| k\right\rangle\langle k|$ and $\rho_{A B}$ is the initial state of Alice and Bob's joint system.

Proof. Initially, Alice and Bob's joint system is in state $\rho_{A B}=\rho_{A} \otimes \rho_{B}$, where $\rho_{A}=\sum_{k=1}^{d} a_{k}|k\rangle\langle k|$ and $\rho_{B}=$ $\sum_{k=1}^{d} b_{k}|k\rangle\langle k|$. Note that we extend $\rho_{A}$ into the space $\mathcal{H}_{d}$ by setting $a_{k}=0$ for $k>d_{A}$. In the following discussion, we denote $a_{k}^{-1}=0$ for $k$ such that $a_{k}=0$. by

According to Lemma 4, the probability $p_{i}$ is upper bounded

$$
p_{i} \leqslant\left\|\rho_{A B}^{-\frac{1}{2}} \rho_{U} \rho_{A B}^{-\frac{1}{2}}\right\|_{\infty}^{-1},
$$

where the inverse operator is defined on the corresponding support space. The equality in Eq. (20) holds when $M_{i}^{T}=$ $\sqrt{p_{i}} \rho_{A B}^{-\frac{1}{2}} \rho_{U}$. A simple calculation shows that $\rho_{A B}^{-\frac{1}{2}}\left|\Omega_{U}\right\rangle=$ $\frac{1}{\sqrt{d}} \sum_{k, t=1}^{d} a_{k}^{-\frac{1}{2}} b_{t}^{-\frac{1}{2}}\langle k|U| t\rangle|k\rangle|t\rangle$. Thus, we get

$$
\begin{aligned}
\left\|\rho_{A B}^{-\frac{1}{2}} \rho_{U} \rho_{A B}^{-\frac{1}{2}}\right\|_{\infty} & =\| \rho_{A B}^{-\frac{1}{2}}\left|\Omega_{U}\right\rangle \|^{2} \\
& =\frac{1}{d} \sum_{k, t=1}^{d} a_{k}^{-1} b_{t}^{-1}\langle k|U| t\rangle\left\langle t\left|U^{\dagger}\right| k\right\rangle \\
& =\frac{1}{d} \operatorname{tr}\left(U \rho_{B}^{-1} U^{\dagger} \rho_{A}^{-1}\right)
\end{aligned}
$$

The eigenvalues of $\rho_{A}^{-1}$ are $a_{d_{A}}^{-1} \geqslant \cdots \geqslant a_{1}^{-1}>a_{d_{A}+1}^{-1}=\cdots=$ $a_{d}^{-1}=0$. Those of $U \rho_{B}^{-1} U^{\dagger}$ are $b_{d}^{-1} \geqslant \cdots \geqslant b_{1}^{-1}>0$. Applying Lemma 5, we get

$$
\operatorname{tr}\left(U \rho_{B}^{-1} U^{\dagger} \rho_{A}^{-1}\right) \geqslant \sum_{k=1}^{d_{A}} \frac{1}{a_{k} b_{d_{A}+1-k}},
$$

where the equality holds when

$$
U=\left(\sum_{k=1}^{d_{A}}\left|d_{A}+1-k\right\rangle\langle k|\right) \oplus I,
$$

where the term in the direct sum acts in the kernel space of $\rho_{A}$.
Therefore, we get the upper bound of the probability $p_{i}$ as

$$
p_{i} \leqslant \frac{d}{\sum_{k=1}^{d_{A}} \frac{1}{a_{k} b_{d_{A}+1-k}}},
$$

where the equality holds when the measurement operator is $M_{i}=\sqrt{p_{\max }}\left|\Omega_{U}\right\rangle\left\langle\Omega_{U}\right| \rho_{A B}^{-\frac{1}{2}}$ and $U$ takes the form defined in Eq. (22).

\section{DISCUSSION AND CONCLUSION}

In this paper, we have exploited the basic configuration of quantum repeaters from the perspectives of both entanglementconcentration rate and LOCC complexity. For the scenario with two different two-qubit pure states, we have constructed a protocol to concentrate entanglement. The protocol is optimal for the perspective entanglement-concentration rate and efficient for the perspective LOCC complexity. We also find a criterion for the projective measurement to achieve the optimal entanglement-concentration rate. For the scenario where general pure states are prepared and general measurements are allowed, we get the upper bound on the probability of a successful measurement outcome which produces a maximally entangled state between Alice and Bob without any further local operations.

The protocol is composed of two steps. First, Clare performs a measurement operation. Second, based on Clare's measurement outcome, Bob chooses the corresponding strategy; namely, Bob does not do any further local operations or performs the corresponding general measurements. In general, the protocol can concentrate entanglement from the scenario with the optimal rate. We reduced the LOCC complexity via the strategy that no further local operation was needed if a maximally entangled state could be created between Alice and Bob when a measurement outcome was observed by Clare.

For the scenario with different two-qubit states $\left|\Phi_{\theta}\right\rangle$ and $\left|\Phi_{\eta}\right\rangle$, we have constructed a projective measurement such that the maximally entangled state could be created after two of the four measurement outcomes. Such measurement outcomes could be observed with probability $\frac{\sin ^{2} 2 \theta \sin ^{2} 2 \eta}{2\left(1-\cos ^{2} 2 \theta \cos ^{2} 2 \eta\right)}$. If the states for the scenario are the same, say $\left|\Phi_{\theta}\right\rangle$, we can construct a projective measurement with three successful projections to produce maximally entangled states without any further local operations. The corresponding probability is $\frac{\sin ^{2} 2 \theta\left(3+\cos ^{2} 2 \theta\right)}{4\left(1+\cos ^{2} 2 \theta\right)}$.

\section{ACKNOWLEDGMENTS}

Z.S. is delighted to thank Prof. Yuan Feng for illuminating and fruitful discussions. This research was partially supported by the China Scholarship Council (Grant No. 201206270069), Australian Research Council (Grant No. DP160101652), and National Natural Science Foundation of China (Grants No. 61472452 and No. 61772565).
[1] M. A. Nielsen and I. L. Chuang, Quantum Computation and Quantum Information (Cambridge University Press, Cambridge, UK, 2000).

[2] M. M. Wilde, Quantum Information Theory (Cambridge University Press, Cambridge, UK, 2013).
[3] C. H. Bennett, G. Brassard, C. Crépeau, R. Jozsa, A. Peres, and W. K. Wootters, Phys. Rev. Lett. 70, 1895 (1993).

[4] P. W. Shor and J. Preskill, Phys. Rev. Lett. 85, 441 (2000).

[5] C. H. Bennett and S. J. Wiesner, Phys. Rev. Lett. 69, 2881 (1992).

[6] Y. Feng, R. Duan, and Z. Ji, Phys. Rev. A 74, 012310 (2006). 
[7] H.-J. Briegel, W. Dur, J. I. Cirac, and P. Zoller, Phys. Rev. Lett. 81, 5932 (1998).

[8] N. Sangouard, C. Simon, H. de Riedmatten, and N. Gisin, Rev. Mod. Phys. 83, 33 (2011).

[9] M. Takeoka, S. Guha, and M. M. Wilde, Nat. Commun. 5, 5235 (2014).

[10] M. Takeoka, K. P. Seshadreesan, and M. M. Wilde, Phys. Rev. Lett. 119, 150501 (2017).

[11] M. M. Wilde, M. Tomamichel, and M. Berta, IEEE Trans. Info. Theory 63, 1792 (2017).

[12] M. M. Wilde, M. Tomamichel, and M. Berta, Phys. Lett. A 373, 2301 (2009).

[13] L. M. Duan, M. D. Lukin, J. I. Cirac, and P. Zoller, Nature 414, 413 (2001).

[14] M. S. Shahriar, G. S. Pati, and K. Salit, Phys. Rev. A 75, 022323 (2007).

[15] A. I. Lvovsky, B. C. Sanders, and W. Tittel, Nat. Photon. 3, 706 (2009).

[16] J. B. Brask, I. Rigas, E. S. Polzik, U. L. Andersen, and A. S. Sørensen, Phys. Rev. Lett. 105, 160501 (2010).

[17] J. Dias and T. C. Ralph, Phys. Rev. A 95, 022312 (2017).

[18] Z. Zhao, T. Yang, Y.-A. Chen, A.-N. Zhang, and J.-W. Pan, Phys. Rev. Lett. 90, 207901 (2003).

[19] A. Kuzmich, W. P. Bowen, A. D. Boozer, A. Boca, C. W. Chou, L.-M. Duan, and H. J. Kimble, Nature 423, 731 (2003).

[20] A. Scherer, B. C. Sanders, and W. Tittel, Opt. Express 19, 3004 (2011).

[21] P. Xu, H. L. Yong, L. K. Chen, C. Liu, T. Xiang, X. C. Yao, H. Lu, Z. D. Li, N. L. Liu, L. Li, T. Yang, C. Z. Peng, B. Zhao,
Y. A. Chen, and J. W. Pan, Phys. Rev. Lett. 119, 170502 (2017).

[22] C.-Y. Lu, T. Yang, and J.-W. Pan, Phys. Rev. Lett. 103, 020501 (2009).

[23] X. Su, C. Tian, X. Deng, Q. Li, C. Xie, and K. Peng, Phys. Rev. Lett. 117, 240503 (2016).

[24] M. Zukowski, A. Zeilinger, M. A. Horne, and A. K. Ekert, Phys. Rev. Lett. 71, 4287 (1993).

[25] C. H. Bennett, H. J. Bernstein, S. Popescu, and B. Schumacher, Phys. Rev. A 53, 2046 (1996).

[26] Y. B. Sheng and L. Zhou, Entropy 15, 1776 (2013).

[27] G. Vidal, Phys. Rev. Lett. 83, 1046 (1999).

[28] Y. Feng, R. Duan, and M. S. Ying, IEEE Trans. Info. Theory 51, 1090 (2005).

[29] B. He and J. A. Bergou, Phys. Rev. A 78, 062328 (2008).

[30] S. Bose, V. Vedral, and P. L. Knight, Phys. Rev. A 57, 822 (1998).

[31] B.-S. Shi, Y.-K. Jiang, and G.-C. Guo, Phys. Rev. A 62, 054301 (2000).

[32] L. Hardy and D. D. Song, Phys. Rev. A 62, 052315 (2000).

[33] S. Perseguers, J. I. Cirac, A. Acín, M. Lewenstein, and J. Wehr, Phys. Rev. A 77, 022308 (2008).

[34] A. Sen(De), U. Sen, Č. Brukner, V. Bužek, and M. Żukowski, Phys. Rev. A 72, 042310 (2005).

[35] Z. Su, Quant. Info. Proc. 16, 28 (2017).

[36] M. M. Wolf, Quantum channels \& operations. Guided tour (2012); https://www-m5.ma.tum.de/foswiki/pub/M5/ Allgemeines/MichaelWolf/QChannelLecture.pdf.

[37] A. S. Lewis, SIAM J. Optim. 6, 164 (1996). 